\title{
Effects of vitamin K2 supplementation on atherogenic status of individuals with type 2 diabetes: a randomized controlled trial
}

\author{
Fatemeh Rahimi Sakak ${ }^{1,2}$, Nazanin Moslehi ${ }^{*}$, Hengameh Abdi ${ }^{3}$ and Parvin Mirmiran ${ }^{1,2}$
}

\begin{abstract}
Background: This study was aimed to examine the effects of vitamin K2 supplementation on atherogenic status, assessed by insulin resistance (IR)-related indexes, in patients with type 2 diabetes mellitus (T2DM).

Methods: In this double-blind, controlled trial, 68 patients with T2DM on the oral glucose-lowering medications were randomly allocated into two groups receiving daily intakes of $360 \mu \mathrm{g}$ MK-7 or placebo for 12 weeks. Eight different IR-related indexes were calculated at the baseline and end of the trial.

Results: At the end of the study, atherogenic coefficient (mean \pm SD: $-0.21 \pm 0.45$ vs. $0.02 \pm 0.43 ; p=0.043$ ), triglyceride-glucose index ( $8.88 \pm 0.55$ vs. $9.23 \pm 0.69 ; p=0.029)$, and atherogenic index of plasma ( $0.37 \pm 0.27$ vs. $0.51 \pm 0.24 ; p=0.031$ ) were significantly lower in the vitamin K2 group, compared to the placebo. However, after accounting for their baseline values, the differences were no more significant. No significant differences were observed in Castelli's I and II risk indexes, the ratio of triglycerides to high-density lipoprotein cholesterol, lipoprotein combine index, and the metabolic score for insulin resistance index between the two groups at the end of the study.
\end{abstract}

Conclusions: Daily intakes of $360 \mu \mathrm{g}$ vitamin K2 in the form of MK-7 for 12 weeks could not improve the IR-related indexes of Cardiovascular Diseases risk.

Trial registration: The trial was registered on Iranian Registry of Clinical Trials registry (Trial ID. IRCT20190824044592N1) on 22 December 2019. The record can be found at https://en.irct.ir/trial/41728.

Keywords: Type 2 diabetes, Vitamin K, Menaquinone-7, Atherogenic status, Atherogenic index, Cardiovascular diseases

\section{Background}

The burden of diabetes on public health and economic status will increase in the next few decades globally by raising the number of patients with diabetes [1]. These patients can live longer than before with medical science advancement, which increases the risk of diabetic-

\footnotetext{
* Correspondence: moslehinazanin@yahoo.com; moslehinazanin@sbmu.ac.ir ${ }^{1}$ Nutrition and Endocrine Research Center, Research Institute for Endocrine Sciences, Shahid Beheshti University of Medical Sciences, No. 24, Shahid Arabi St, Yemen Blvd, Chamran Exp, Tehran 1985717413, Iran Full list of author information is available at the end of the article
}

related complications [2]. Patients with diabetes are actually at risk of cardiovascular diseases (CVD), about four-time more than healthy ones, and the risk of cardiovascular deaths is also noticeably high [3]. Insulin resistance (IR) through glucose and lipid metabolism impairments is one of the leading causes of CVD risk [4]. The role of IR in endothelial dysfunction, plaque formation and progression, and vascular calcification is also indicated [4, 5]. Different indexes have been developed to assess IR indirectly, such as the atherogenic index of plasma (AIP), lipoprotein combine index (LCI), the

C C The Author(s). 2021 Open Access This article is licensed under a Creative Commons Attribution 4.0 International License, which permits use, sharing, adaptation, distribution and reproduction in any medium or format, as long as you give appropriate credit to the original author(s) and the source, provide a link to the Creative Commons licence, and indicate if changes were made. The images or other third party material in this article are included in the article's Creative Commons licence, unless indicated otherwise in a credit line to the material. If material is not included in the article's Creative Commons licence and your intended use is not permitted by statutory regulation or exceeds the permitted use, you will need to obtain permission directly from the copyright holder. To view a copy of this licence, visit http://creativecommons.org/licenses/by/4.0/ The Creative Commons Public Domain Dedication waiver (http://creativecommons.org/publicdomain/zero/1.0/) applies to the data made available in this article, unless otherwise stated in a credit line to the data. 
metabolic score for insulin resistance index (METSIR), and triglyceride-glucose index (TyG-Index). These non-insulin based indexes of IR can be easily determined by combinations of traditional risk factors of CVD, including fasting plasma glucose (FPG), lipid profiles, and body mass index (BMI). These indexes were associated with carotid atherosclerosis, vascular calcification, risk of CVD, and clinical outcomes, and therefore suggested to be useful for early detection of subclinical atherosclerosis [5-8]. The indexes are proposed to predict CVD events stronger than the routine individual lipid measurements [9].

A review article recently provided plausible evidence, suggesting the protective roles of vitamin $\mathrm{K}$ against vascular calcification through its anti-inflammatory effects and carboxylation of vitamin K-dependent protein (VKDP). Gamma carboxylation of Matrix Gla protein (MGP), a vitamin K-dependent protein, inhibits vascular mineralization. Of three forms of MGP identified in blood, dephosphorylated-uncarboxylated MGP (dpucMGP), a biomarker for vitamin $\mathrm{K}$ deficiency, was positively associated with vascular calcification, CVD events, and mortality [10]. However, a systematic review of the effects of vitamin $\mathrm{K}$ supplementation on clinical measures of vascular calcification, atherosclerosis, and arterial stiffness, reported that while the supplementation can decrease the levels of dp-ucMGP, the beneficial effects of vitamin $\mathrm{K}$ on reducing the risk of CVD is uncertain, according to the results of the current studies. Of the nine studies included in the systematic review, one conducted among patients with type 2 diabetes mellitus (T2DM) [11]. Vitamin K and CVD risks in patients with T2DM deserve further investigations because of the lower levels of vitamin $K$ in the blood [12] and higher susceptibility to vascular calcification in this cohort of patients than healthy ones [13]. In a randomized clinical trial, we could find significant improvements in glycemic measures of FPG and glycated hemoglobin (HbA1c) in patients with T2DM with vitamin K2 supplementation in the form of menaquinone-7 (MK-7), compared to the placebo [14]. In this study, we aimed to investigate whether the MK-7 supplementation at a dose of $360 \mu \mathrm{g} /$ d for 12 weeks could affect atherogenic status in patients with T2DM, assessed by eight different IR-related indexes, as secondary analyses.

\section{Materials and methods Participants}

The original study design, which aimed to assess the effects of MK-7 on FPG and HbA1c in participants with diabetes, was reported in detail previously [14]. Briefly, in this randomized, double-blind, and placebo-controlled clinical trial, 68 patients with diabetes medicated with oral hypoglycemic drugs, aged $30-70$, had at least one- year history of diabetes, and HbA1c between 6.5 and $10 \%$, participated. Pregnant and lactating women, participants on insulin therapy or medications affecting the metabolism of vitamin $\mathrm{K}$, and those with a history of chronic diseases such as heart, liver, kidney, and cancer were excluded.

\section{Ethical issues}

This trial was designed according to the Declaration of Helsinki principles; the Ethics Committee of the Research Institute for Endocrine Sciences at the Shahid Beheshti University of Medical Sciences confirmed the protocols of the trial (IR.SBMU.ENDOCRINE.REC.1399.141). This study was also registered in the Iranian registry of clinical trials (Identifier number: IRCT20190824044592N1; date of registration: 22 December 2019). Before the study enrolment, the purpose and possible risks of the trial procedure were clearly explained to the participants, and then written informed consent was obtained from them.

\section{Trial design}

After meeting the inclusion criteria, 68 eligible patients referred to three diabetic clinics in the north of Tehran were randomly assigned into the treatment groups of vitamin K2 $(n=34)$ or placebo $(n=34)$ using the method of block randomization with the block size of four and allocation ratio of 1:1. Each patient received a capsule container of vitamin $\mathrm{K} 2$ or placebo monthly in this trial. They were instructed to take two capsules of vitamin K2 $(180 \mu \mathrm{g}$ MK-7) or placebo per day after breakfast and dinner meals for 12 weeks. All participants were asked to avoid any dietary intake or physical activity changes throughout the intervention period and avoid consuming any other nutritional supplements. For designing a double-blinded trial, both vitamin K2 and placebo capsules were made in the same appearance and packaging by Arian Salamat Sina Pharmaceutical Company (Tehran, Iran). Microcrystalline Cellulose was used to fill the placebo capsules. At the Research Institute for Endocrine Sciences, a study leader generated the random assignment sequence, but she did not involve recruitment and trial procedures. The allocation was concealed by sequentially numbered of the capsules containers. A study executor enrolled the participants and assigned them to the intervention groups. Treatment assignments were blinded to all participants and the study executers until the statistical analyses were done.

At the end of each month during the study, participants received a new container of capsules and returned the previous one. Compliance with the treatment was checked by counting the remaining pills in each box. Moreover, we made weekly telephone calls to assess 
their adherence to the study protocol and the trial's possible side effects.

\section{Biological variables analysis}

After the 10-h overnight fasting, a $5 \mathrm{ml}$ blood sample was collected at the beginning and end of the study at the laboratory of the Research Institute for Endocrine Sciences at Shahid Beheshti University of Medical Sciences. The enzymatic colorimetric method was used to measure FPG and Triglyceride levels by Parsazmun kits, Tehran, Iran. The enzymatic photometric method was used to assess the low-density lipoprotein cholesterol (LDL-C), high-density lipoprotein cholesterol (HDL-C), and total cholesterol levels by Parsazmun kits, Tehran, Iran. Intra-assay coefficients of variations $(\mathrm{CVs})$ were $0.86 \%$ for FPG, $1.98 \%$ for total cholesterol, $1.46 \%$ for LDL-C, $0.61 \%$ for HDL-C, and $2.42 \%$ for triglycerides. The following indexes were calculated:

1. Castelli's risk index $I=\frac{\text { Total cholesterol }}{H D L-C}$ [15]

2. $\frac{\text { Triglyceride }}{H D L-C}[16]$

3. Atherogenic coefficient $(A C)=\frac{\text { Non } H D L-C}{H D L-C}$ [17]

4. Castelli's risk index II $=\frac{L D L-C}{H D L-C}[15]$

5. Triglyceride-glucose index $($ TyG-Index $)=\ln$ $\frac{F P G \times \text { Triglyceride }}{2}[18]$

6. Atherogenic index of plasma $(A I P)=\log \frac{\text { Triglyceride }}{H D L-C}$ [19]

7. Metabolic score for insulin resistance index (METS $-I R)=\frac{(L N((2 \times F P G)+\text { Triglyceride }) \times B M I)}{(L N(H D L-C))}[20]$

8. Lipoprotein combine index $(L C I)=$ Total cholesterol $\times$ Triglyceride $\times L D L-C$ $H D L-C$

\section{Other clinical variables analysis}

Data on disease duration, demographic variables, and drug medications were collected by a designed questionnaire. Weight and height were measured, and BMI was calculated [14]. Physical activity and dietary intakes were assessed at baseline and end of the trial using the short form of the International Physical Activity Questionnaires (IPAQ) and three 24-h food recalls, respectively.

\section{Statistical analysis}

The study's sample size was calculated based on the primary outcomes of the study [14]. The SPSS version 21 software was used to determine the statistical analyses. The normality of the variables was checked by the Kolmogorov-Smirnov test. Baseline characteristics of participants were compared between the two groups of the vitamin $\mathrm{K} 2$ and the placebo using the Student's ttest for normally distributed variables, the Mann-Whitney $U$ test for non-normally distributed variables, and $\chi^{2}$ test for qualitative variables. The mean value of each IRrelated index of CVD risk after supplementation compared to the baseline values within each group using the paired sample t-test. The Student's t-test was also used to compare each IR-related index's mean value before and after supplementation between the two groups. Analysis of Covariance (ANCOVA) was used to adjust the baseline value of each outcome. The natural logtransformation was performed for non-normally distributed variables of AC and LCI before statistical analyses; mean and standard deviation (SD) values were reported for the natural log-transformed.

\section{Results \\ Participants' baseline characteristics}

Participants were recruited from October 2019 to January 2020, and the follow-up was ended in April 2020. Of 68 participants included, 63 completed the study (32 patients in the vitamin $\mathrm{K} 2$ group, 31 patients in the placebo group); two individuals in the vitamin $\mathrm{K} 2$ group and three individuals in the placebo groups withdrew. The reasons for withdrawal were personal reasons $(n=$ $3)$, immigration $(n=1)$, and bone surgery $(n=1)$. At the baseline, the mean age, diabetes duration, weight, BMI, and physical activity did not significantly differ between the two groups. Moreover, no significant difference was found in the number of females, smokers, and academic education between the vitamin $\mathrm{K} 2$ and the placebo groups. Baseline values of the indexes were not significantly different between the two groups (Table 1).

Of the participants, $64.7 \%$ were treated with lipidlowering drugs, $97 \%$ were treated with metformin, and $19.2 \%$ were treated with the sodium-glucose cotransporter-2 (SGLT2) inhibitors. At the baseline, the frequency of lipid-lowering and oral hypoglycemic drugs did not significantly differ between the two groups.

Physical activity at the end of the trial and dietary intakes at baseline and end of the study were not significantly different between the two groups (data were not shown).

\section{Effects of vitamin $\mathrm{K}_{2}$ on atherogenic status}

Compared to the baseline, a marginally significant reduction was observed in the METS-IR in the vitamin K2 group (mean change from baseline: $-0.80 \pm 2.24 ; p=$ 0.052 ). None of the other indexes changed significantly compared to the baseline within groups. At the end of the study, AC $(-0.21 \pm 0.45$ vs. $0.02 \pm 0.43 ; p=0.043)$, TyG (8.88 \pm 0.55 vs. $9.23 \pm 0.69 ; p=0.029)$, and AIP $(0.37 \pm 0.27$ vs. $0.51 \pm 0.24 ; p=0.031)$ were significantly lower in the vitamin $\mathrm{K} 2$ group compared to the placebo group, but the differences became non-significant after adjusting for their baseline values. The other indexes were not significantly different between the two groups at the end of the study (Table 2). 
Table 1 Baseline characteristics of the participants

\begin{tabular}{llll}
\hline Variables & Vitamin $\mathbf{K}_{\mathbf{2}}$ group & Placebo group & $\boldsymbol{P}_{\text {-value }} \mathbf{2}^{2}$ \\
\hline Number & 32 & 31 & - \\
Age, years & $56.8 \pm 7.34$ & $58.2 \pm 7.39$ & 0.440 \\
Disease duration, years & $7.81 \pm 4.27$ & $9.68 \pm 5.93$ & 0.156 \\
Female, n (\%) & $21(65.6 \%)$ & $18(58.1 \%)$ & 0.609 \\
Smokers, n (\%) & $5(15.6 \%)$ & $2(6.5 \%)$ & 0.426 \\
Academic education, n (\%) & $7(21.9 \%)$ & $14(45.1 \%)$ & 0.201 \\
Weight, kg & $7.29 \pm 14.7$ & $74.9 \pm 16.7$ & 0.607 \\
Body mass index, kg/m ${ }^{2}$ & $27.9 \pm 4.90$ & $27.5 \pm 3.60$ & 0.729 \\
Physical activity, Met-min/week & $425(209-679)$ & $834(240-1386)$ & 0.063 \\
Atherogenicity index & & & 0.133 \\
Castelli index I & $3.33 \pm 0.79$ & $3.65 \pm 0.89$ & 0.194 \\
Triglycerides / HDL-C ratio & $1.33 \pm 0.85$ & $1.64 \pm 1.06$ & 0.124 \\
AC ${ }^{3}$ & $-0.17 \pm 0.48$ & $0.01 \pm 0.44$ & 0.308 \\
Castelli index II & $1.68 \pm 0.48$ & $1.80 \pm 0.51$ & 0.172 \\
TyG-Index & $9.02 \pm 0.56$ & $9.23 \pm 0.64$ & 0.109 \\
AlP & $0.40 \pm 0.27$ & $0.51 \pm 0.24$ & 0.576 \\
METS-IR & $43.9 \pm 9.09$ & $45.1 \pm 7.14$ & 0.382 \\
LCP & $1.95 \pm 0.37$ & $2.03 \pm 0.40$ &
\end{tabular}

AC Atherogenic Coefficient, AIP Atherogenic Index of Plasma, LCI Lipoprotein Combine Index, METS-IR Metabolic Score for Insulin Resistance, TyG-Index Triglyceride glucose Index

${ }^{1}$ Data are presented as mean $\pm \mathrm{SD}$, median (quartile 1-quartile 3), and number (\%)

${ }^{2}$ Based on T-test for normally-distributed, Mann-Whitney U-test for non-normally distributed and chi-square for categorical variables

${ }^{3}$ Natural log-transformed values are reported

\section{Sensitivity analysis}

Glucose-lowering medications of eight participants were changed during the study, all of whom were in the placebo group (drug dose reduction $(n=4)$, dose increment $(n=3)$, added insulin $(n=1))$. Lipid-lowering medications did not change during the study. However, no remarkable differences were observed in our findings after re-analyzing the data by excluding the eight participants.

\section{Compliance and tolerability}

The study's compliance was $95.9 \pm 5.99 \%$; none of the participants consumed less than $70 \%$ of the study capsules. Vitamin K2 and placebo capsules were both welltolerated, and none of the participants reported any side effects related to the capsules' consumption.

\section{Discussion}

After a 12-week supplementation of $360 \mu \mathrm{g} /$ day MK-7, AC, AIP, and TyG index were significantly lower in the vitamin $\mathrm{K} 2$ group than the placebo group, but the differences did not remain significant after accounting for their baseline values. No significant differences were observed in the other indexes within and between groups.

Vitamin $\mathrm{K}$ involves different physiologic activities by promoting the carboxylation process of VKDP as a cofactor for the enzyme of gamma-carboxylase. Gamma- carboxylation of the proteins makes them biologically active [22]. The most well-known VKDP produced by smooth muscle cells is MGP that inhibits vascular calcification. A higher circulatory level of dp-ucMPG, the best vitamin $\mathrm{K}$ insufficiency marker, has been related to a higher risk of vascular calcification, cardiovascular events, and CVD mortality $[10,23]$. The antiinflammatory effects of vitamin $\mathrm{K}$ also increase the possibility of its contribution to cardiovascular health [10, 22]. Higher intakes of dietary vitamin $\mathrm{K}$ have been associated with the lower risk of total coronary heart disease based on the pooled data from prospective studies on associations between dietary intakes of vitamin K1 $(n=4)$ and $\mathrm{K} 2(n=2)$ and cardiovascular events. However, no significant association between dietary intakes of vitamin $\mathrm{K}$ and CVD mortality and stroke was found [23]. A small number of clinical trials examined the effects of vitamin K supplementation on CVD markers [11]. Recently, a study systematically reviewed clinical trials' findings for the effects of vitamin $\mathrm{K}$ supplementation on the clinical proxy of CVD. Vitamin K supplementation could reduce dp-ucMPG significantly compared to the placebo in 7 out of 9 studies, but the effects of the supplementation on vascular calcification, atherosclerosis, and arterial stiffness were not convincing due to the scarcity of the studies [11]. 
Table 2 The effects of vitamin K2 supplementation on atherogenic indexes ${ }^{1}$

\begin{tabular}{|c|c|c|c|c|}
\hline Variables & Vitamin K2 group $(n=32)$ & Placebo group $(n=31)$ & Between group $p$-value $e^{3}$ & Adjusted $p$-value ${ }^{4}$ \\
\hline \multicolumn{5}{|l|}{$\overline{C \text { Castelli index }\left.\right|^{2}}$} \\
\hline After 12-week & $3.29 \pm 0.75$ & $3.67 \pm 0.91$ & 0.073 & 0.333 \\
\hline Mean change from baseline & $-0.04 \pm 0.45$ & $0.02 \pm 0.55$ & 0.657 & - \\
\hline Within group $P$-value ${ }^{5}$ & 0.657 & 0.834 & - & - \\
\hline \multicolumn{5}{|l|}{ Triglycerides / HDL-C ratio } \\
\hline After 12-week & $2.81 \pm 1.94$ & $3.74 \pm 2.25$ & 0.083 & 0.252 \\
\hline Mean change from baseline & $-0.10 \pm 0.71$ & $-0.01 \pm 0.51$ & 0.570 & - \\
\hline Within group $P$-value ${ }^{5}$ & 0.442 & 0.925 & - & - \\
\hline \multicolumn{5}{|l|}{$A C^{6,7}$} \\
\hline After 12-week & $-0.21 \pm 0.45$ & $0.02 \pm 0.43$ & 0.043 & 0.167 \\
\hline Mean change from baseline & $-0.03 \pm 0.25$ & $0.01 \pm 0.21$ & 0.407 & - \\
\hline Within group $P$-value 5 & 0.440 & 0.709 & - & - \\
\hline \multicolumn{5}{|l|}{ Castelli index $\|^{8}$} \\
\hline After 12-week & $1.69 \pm 0.41$ & $1.88 \pm 0.54$ & 0.132 & 0.264 \\
\hline Mean change from baseline & $0.02 \pm 0.30$ & $0.07 \pm 0.38$ & 0.506 & - \\
\hline Within group $P$-value $e^{5}$ & 0.777 & 0.297 & - & - \\
\hline \multicolumn{5}{|l|}{ TyG-Index } \\
\hline After 12-week & $8.88 \pm 0.55$ & $9.23 \pm 0.69$ & 0.029 & 0.084 \\
\hline Mean change from baseline & $-0.14 \pm 0.46$ & $0.0005 \pm 0.42$ & 0.205 & - \\
\hline Within group $P$-value ${ }^{5}$ & 0.091 & 0.995 & - & - \\
\hline \multicolumn{5}{|l|}{ AIP } \\
\hline After 12-week & $0.37 \pm 0.27$ & $0.51 \pm 0.24$ & 0.031 & 0.135 \\
\hline Mean change from baseline & $-0.03 \pm 0.15$ & $0.003 \pm 0.12$ & 0.325 & - \\
\hline Within group $P$-value ${ }^{5}$ & 0.245 & 0.901 & - & - \\
\hline \multicolumn{5}{|l|}{ METS-IR } \\
\hline After 12-week & $43.1 \pm 8.42$ & $45.2 \pm 7.29$ & 0.292 & 0.067 \\
\hline Mean change from baseline & $-0.80 \pm 2.24$ & $0.15 \pm 2.30$ & 0.101 & - \\
\hline Within group $P$-value ${ }^{5}$ & 0.052 & 0.719 & - & - \\
\hline \multicolumn{5}{|l|}{$\mathrm{LCl}^{7}$} \\
\hline After 12-week & $1.91 \pm 0.34$ & $2.04 \pm 0.43$ & 0.194 & 0.315 \\
\hline Mean change from baseline & $1.34 \pm 0.57$ & $1.54 \pm 0.53$ & 0.167 & - \\
\hline Within group $P$-value ${ }^{5}$ & 0.391 & 0.842 & - & - \\
\hline
\end{tabular}

AC Atherogenic Coefficient, AIP Atherogenic Index of Plasma, LCI Lipoprotein Combine Index, METS-IR Metabolic Score for Insulin Resistance, TyG-Index Triglyceride glucose Index, HDL-C High-density lipoprotein cholesterol

${ }^{1}$ Data are presented as mean \pm SD

${ }^{2}$ Total cholesterol / HDL-C ratio

${ }^{3}$ Based on independent sample T-test

${ }^{4}$ Based on Analysis of Covariance (ANCOVA)

${ }^{5}$ Based on paired sample T-test

${ }^{6}$ Non-HDL-C / HDL-C ratio

${ }^{7}$ Natural log-transformed values are reported

${ }^{8} \mathrm{LDL}-\mathrm{C} / \mathrm{HDL}-\mathrm{C}$ ratio

Circulatory vitamin $\mathrm{K} 1$ was lower in patients with T2DM compared to non-diabetic individuals. The lower vitamin $\mathrm{K}$ status was also correlated with higher vascular inflammation as measured by monocyte chemoattractant protein-1 (MCP-1) [12]. Increased vascular calcification is also documented in patients with T2DM due to their inflammatory state. Moreover, inadequate control of glycemia, increased IR, and kidney disease occurrence accelerates the calcification process [24]. Increased risk of CVD, peripheral arterial disease, and heart failure in a prospective study with a median duration of 11.2 years were reported for patients with T2DM with low vitamin 
K status, as assessed by plasma dp-ucMGP [25]. To the best of our knowledge, the effects of vitamin K supplementation on the vasculature in patients with T2DM has been investigated only in one clinical trial. The study examined the effect of MK-7 supplementation on the clinical surrogate of artery calcification measured by computed tomography [26]. Based on the findings, sixmonth supplementation of $360 \mu \mathrm{g} / \mathrm{d}$ MK-7 in patients with T2DM and pre-existing CVD did not decrease significantly systemic arterial calcification and calcification of femoral arteries [26, 27].

Different indexes have been developed to assess IR without a direct measure of insulin concentration. The indexes can be easily determined by routine measurements of FPG, triglycerides, and other lipoproteins. The indexes' associations with subclinical atherosclerosis measurements of coronary artery calcification, arterial stiffness, and vascular flow limitations have been well indicated $[5-9,28]$. The beneficial effects of an intervention on CVD risk by improving the metabolic variables of glucose and lipids can be exerted earlier by determining the indexes rather than atherosclerosis's clinical measurements. Based on our findings, none of the indexes was significantly reduced following MK-7 supplementation for 12 weeks in our participants compared to the placebo. Previously, no studies examined the effects of vitamin $\mathrm{K}$ supplementation on atherogenic indexes.

Randomized double-blinded controlled design, high adherence to the study protocol, using the most effective and bioavailable forms of vitamin $\mathrm{K}$ are strengths of this study. The limitations of our study were: 1) the individuals' responses to vitamin $\mathrm{K}$ supplementation may differ based on their vitamin $\mathrm{K}$ status before the supplementation. However, no measurement has been done for circulatory markers of vitamin $\mathrm{K}$ status in this study, so the participants' vitamin $\mathrm{K}$ status was unclear at the beginning of the study; 2) we had no objective measurement to confirm the compliance of the study assessed by pill counting; 3) most participants were at the normal range of lipid profiles with/without lipid-lowering medications in this study. Therefore, they may have a lower response to the supplementation than those with abnormal lipid profiles. However, the AIP values of $84.1 \%$ of our participants were more than 0.21 , suggesting that they were at a high risk of CVD [9]; 4) this study's sample size is calculated based on the primary outcomes of glycemic variables, so it may not be adequately powered to detect changes in IR-related indexes; 5) testing multiple hypotheses simultaneously causes multiplicity concerns, which may increase the probability of false-positive findings $[29,30]$. However, multiplicity adjustment was not considered in this study because of null findings; 6) the dose of vitamin $\mathrm{K} 2$ and the study duration was determined according to a study's findings suggesting vitamin
K status improvements following $360 \mu \mathrm{g} / \mathrm{d}$ MK-7 supplementations for 12 weeks [11]. However, this dose and duration may not be adequate to observe any significant effects on atherogenic status.

\section{Conclusion}

In conclusion, our findings could not show any significant improvement in the atherogenic status of patients with T2DM as measured by IR-related indexes of CVD risk, including Castelli indexes, triglycerides/HDL-C ratio, AC, TyG-Index, AIP, METS-IR, and LCI, after 12week supplementation of $360 \mu \mathrm{g} / \mathrm{d}$ MK-7. More clinical trials are needed to clarify the effects of MK-7 supplementation on laboratory or clinical markers of CVD in patients with T2DM.

\section{Abbreviations}

AC: Atherogenic coefficient; AIP: Atherogenic index of plasma; ANCOVA: Analysis of Covariance; BMI: Body mass index; CVD: Cardiovascular diseases; CVs: Coefficients of variations; Dp-ucMGP: Dephosphorylateduncarboxylated MGP; FPG: Fasting plasma glucose; HbA1c: Glycated hemoglobin; HDL-C: High-density lipoprotein cholesterol; IR: Insulin resistance; IPAQ: International Physical Activity Questionnaires;

LCI: Lipoprotein combine index; LDL-C: Low-density lipoprotein cholesterol; METS-IR: Metabolic score for insulin resistance index; MK-7: Menaquinone-7; MCP-1: Monocyte Chemoattractant Protein-1; MGP: Matrix Gla protein; SD: Standard deviation; SGLT2: Sodium-glucose co-transporter-2; T2DM: Type 2 diabetes mellitus; TyG-Index: Triglyceride-glucose index; VKDP: Vitamin Kdependent protein

\section{Acknowledgements}

We would like to express our sincere appreciation to the trial participants for their cooperation and partnership.

\section{Authors' contributions}

All authors contributed to the study's conceptual design, reviewed and approved the final version of the manuscript. FRS and NM contributed to the data collection, data analyses and interpretation, drafted the manuscript. PM supervised performing the trial. The intellectual comments were provided by $\mathrm{PM}$ and $\mathrm{HA}$.

\section{Funding}

This study was supported by a grant by the Research Institute of Endocrine Sciences, Shahid Beheshti University Medical Sciences, Tehran, Iran.

\section{Availability of data and materials}

All data are presented within the manuscript. Raw data can be available by corresponding authors per reasonable request.

\section{Declarations}

\section{Ethics approval and consent to participate}

This trial was designed according to the Declaration of Helsinki principles; the Ethics Committee of the Research Institute for Endocrine Sciences at the Shahid Beheshti University of Medical Sciences confirmed the protocols of the trial (IR.SBMU.ENDOCRINE.REC.1399.141). Before the study enrolment, the purpose and possible risks of the trial procedure were clearly explained to the participants, and then written informed consent was obtained from them.

\section{Consent for publication}

Not applicable.

Competing interests

The authors declare no conflicts of interest. 


\section{Author details}

Nutrition and Endocrine Research Center, Research Institute for Endocrine Sciences, Shahid Beheshti University of Medical Sciences, No. 24, Shahid Arabi St, Yemen Blvd, Chamran Exp, Tehran 1985717413, Iran. ${ }^{2}$ Department of Clinical Nutrition and Dietetics, Faculty of Nutrition and Food Technology, National Nutrition and Food Technology Research Institute, Shahid Beheshti University of Medical Sciences, Tehran, Iran. ${ }^{3}$ Endocrine Research Center, Research Institute for Endocrine Sciences, Shahid Beheshti University of Medical Sciences, Tehran, Iran.

\section{Received: 26 January 2021 Accepted: 15 April 2021}

\section{Published online: 01 May 2021}

\section{References}

1. Lin X, Xu Y, Pan X, X J J, Ding Y, Sun X, et al. Global, regional, and national burden and trend of diabetes in 195 countries and territories: an analysis from 1990 to 2025. Sci Rep. 2020;10(1):14790. https://doi.org/10.1038/s41 598-020-71908-9.

2. Deshpande AD, Harris-Hayes M, Schootman M. Epidemiology of diabetes and diabetes-related complications. Phys Ther. 2008:88(11):1254-64. https:// doi.org/10.2522/ptj.20080020

3. Barr EL, Zimmet PZ, Welborn TA, Jolley D, Magliano DJ, Dunstan DW, et al. Risk of cardiovascular and all-cause mortality in individuals with diabetes mellitus, impaired fasting glucose, and impaired glucose tolerance: the Australian Diabetes, Obesity, and Lifestyle Study (AusDiab). Circulation. 2007; 116(2):151-7. https://doi.org/10.1161/CIRCULATIONAHA.106.685628.

4. Ormazabal V, Nair S, Elfeky O, Aguayo C, Salomon C, Zuñiga FA. Association between insulin resistance and the development of cardiovascular disease. Cardiovasc Diabetol. 2018;17(1):1-14. https://doi.org/10.1186/s12933-0180762-4.

5. Park K, Ahn CW, Lee SB, Kang S, Nam JS, Lee BK, et al. Elevated TyG index predicts progression of coronary artery calcification. Diabetes Care. 2019; 42(8):1569-73. https://doi.org/10.2337/dc18-1920.

6. Mahat RK, Singh N, Rathore V, Gupta A, Shah RK. Relationship between atherogenic indices and carotid intima-media thickness in prediabetes: a cross-sectional study from Central India. Med Sci. 2018;6(3):55. https://doi. org/10.3390/medsci6030055.

7. Won K-B, Park G-M, Lee S-E, Cho I-J, Kim HC, Lee BK, et al. Relationship of insulin resistance estimated by triglyceride glucose index to arterial stiffness. Lipids Health Dis. 2018;17(1):268. https://doi.org/10.1186/s12944-018-0914-2.

8. Bello-Chavolla OY, Antonio-Villa NE, Vargas-Vázquez A, Martagón AJ, Mehta $\mathrm{R}$, Arellano-Campos $\mathrm{O}$, et al. Prediction of incident hypertension and arterial stiffness using the non-insulin-based metabolic score for insulin resistance (METS-IR) index. J Clin Hypertens (Greenwich). 2019;21(8):1063-70. https:// doi.org/10.1111/jch.13614.

9. Fernández-Macías JC, Ochoa-Martínez AC, Varela-Silva JA, Pérez-Maldonado IN. Atherogenic index of plasma: novel predictive biomarker for cardiovascular illnesses. Arch Med Res. 2019;50(5):285-94. https://doi.org/1 0.1016/j.arcmed.2019.08.009.

10. Shioi A, Morioka T, Shoji T, Emoto M. The inhibitory roles of vitamin $\mathrm{K}$ in progression of vascular calcification. Nutrients. 2020;12(2):583. https://doi org/10.3390/nu12020583.

11. Vlasschaert C, Goss CJ, Pilkey NG, McKeown S, Holden RM. Vitamin K supplementation for the prevention of cardiovascular disease: where is the evidence? A systematic review of controlled trials. Nutrients. 2020;12(10): 2909. https://doi.org/10.3390/nu12102909.

12. Dihingia A, Ozah D, Baruah PK, Kalita J, Manna P. Prophylactic role of vitamin $\mathrm{K}$ supplementation on vascular inflammation in type 2 diabetes by regulating the NF-KB/Nrf2 pathway via activating Gla proteins. Food Funct. 2018;9(1):450-62. https://doi.org/10.1039/c7fo01491k.

13. Kramer CK, Zinman B, Gross JL, Canani LH, Rodrigues TC, Azevedo MJ, et al. Coronary artery calcium score prediction of all cause mortality and cardiovascular events in people with type 2 diabetes: systematic review and meta-analysis. BMJ. 2013;346(mar25 1). https://doi.org/10.1136/bmj.f1654.

14. Rahimi Sakak F, Moslehi N, Niroomand M, Mirmiran P. Glycemic control improvement in individuals with type 2 diabetes with vitamin $\mathrm{K} 2$ supplementation: a randomized controlled trial. Eur J Nutr. 2020. https://doi. org/10.1007/s00394-020-02419-6.

15. Bhardwaj S, Bhattacharjee J, Bhatnagar M, Tyagi S. Atherogenic index of plasma, Castelli risk index and atherogenic coefficient-new parameters in assessing cardiovascular risk. Int J Pharm Biol Sci. 2013;3(3):359-64.
16. Gaziano JM, Hennekens $\mathrm{CH}$, O'Donnell CJ, Breslow JL, Buring JE. Fasting triglycerides, high-density lipoprotein, and risk of myocardial infarction. Circulation. 1997;96(8):2520-5. https://doi.org/10.1161/01.cir.96.8.2520.

17. Olamoyegun MA, Oluyombo R, Asaolu SO. Evaluation of dyslipidemia, lipid ratios, and atherogenic index as cardiovascular risk factors among semiurban dwellers in Nigeria. Ann Afr Med. 2016;15(4):194-9. https://doi.org/1 0.4103/1596-3519.194280.

18. Lambrinoudaki I, Kazani MV, Armeni E, Georgiopoulos G, Tampakis K, Rizos $D$, et al. The TyG index as a marker of subclinical atherosclerosis and arterial stiffness in lean and overweight postmenopausal women. Heart Lung Circ. 2018;27(6):716-24. https://doi.org/10.1016/j.hlc.2017.05.142.

19. Onat A, Can G, Kaya $H$, Hergenç G. "Atherogenic index of plasma" ( $\log 10$ triglyceride/high-density lipoprotein - cholesterol) predicts high blood pressure, diabetes, and vascular events. J Clin Lipidol. 2010;4(2):89-98. https://doi.org/10.1016/j.jacl.2010.02.005.

20. Bello-Chavolla OY, Almeda-Valdes P, Gomez-Velasco D, Viveros-Ruiz T, CruzBautista I, Romo-Romo A, et al. METS-IR, a novel score to evaluate insulin sensitivity, is predictive of visceral adiposity and incident type 2 diabetes. Eur J Endocrinol. 2018;178(5):533-44. https://doi.org/10.1530/EJE-17-0883.

21. Oguntola SO, Hassan MO, Duarte R, Dix-Peek T, Dickens C, Olorunfemi G, et al. Atherosclerotic vascular disease and its correlates in stable black south African kidney transplant recipients. Int J Nephrol Renovasc Dis. 2018;11: 187-93. https://doi.org/10.2147/JNRD.S160553.

22. Palmer CR, Blekkenhorst LC, Lewis JR, Ward NC, Schultz CJ, Hodgson JM, et al. Quantifying dietary vitamin $\mathrm{K}$ and its link to cardiovascular health: a narrative review. Food Funct. 2020;11(4):2826-37. https://doi.org/10.1039/ c9fo02321f.

23. Chen H-G, Sheng L-T, Zhang Y-B, Cao A-L, Lai Y-W, Kunutsor SK, et al. Association of vitamin $\mathrm{K}$ with cardiovascular events and all-cause mortality: a systematic review and meta-analysis. Eur J Nutr. 2019;58(6):2191-205. https://doi.org/10.1007/s00394-019-01998-3.

24. Al-Aly Z. Medial vascular calcification in diabetes mellitus and chronic kidney disease: the role of inflammation. Cardiovasc Hematol Disord Drug Targets. 2007;7(1):1-6. https://doi.org/10.2174/187152907780059047.

25. Dalmeijer GW, van der Schouw YT, Vermeer C, Magdeleyns EJ, Schurgers $L$ Beulens JW. Circulating matrix Gla protein is associated with coronary artery calcification and vitamin K status in healthy women. J Nutr Biochem. 2013; 24(4):624-8. https://doi.org/10.1016/j.jnutbio.2012.02.012.

26. Zwakenberg S, De Jong P, Bartstra J, Van Asperen R, Westerink J, De Valk H, et al. The effect of menaquinone-7 supplementation on vascular calcification in patients with diabetes: a randomized, double-blind, placebocontrolled trial. Am J Clin Nutr. 2019;110(4):883-90. https://doi.org/10.1093/a $\mathrm{jcn} / \mathrm{nqz} 147$

27. Bartstra JW, Draaisma F, Zwakenberg SR, Lessmann N, Wolterink JM, van der Schouw $\mathrm{YT}$, et al. Six months vitamin $\mathrm{K}$ treatment does not affect systemic arterial calcification or bone mineral density in diabetes mellitus 2. Eur J Nutr. 2020;60(3):1-9. https://doi.org/10.1007/s00394-020-02412-z.

28. Sánchez-lñigo L, Navarro-González D, Fernández-Montero A, PastranaDelgado J, Martínez JA. The TyG index may predict the development of cardiovascular events. Eur J Clin Investig. 2016;46(2):189-97. https://doi.org/1 0.1111/eci.12583.

29. Li G, Taljaard M, Van den Heuvel ER, Levine MA, Cook DJ, Wells GA, et al. An introduction to multiplicity issues in clinical trials: the what, why, when and how. Int J Epidemiol. 2017;46(2):746-55. https://doi.org/10.1093/ije/dyw320.

30. Dmitrienko A, D'Agostino RB Sr. Multiplicity considerations in clinical trials. N Engl J Med. 2018;378(22):2115-22. https://doi.org/10.1056/NEJMra1709701.

\section{Publisher's Note}

Springer Nature remains neutral with regard to jurisdictional claims in published maps and institutional affiliations. 